\title{
Critical angle for reflection at a liquid-solid interface in single crystals
}

\author{
Edmund G. Henneke II and Gerald L. Jones* \\ Department of Engineering Science and Mechanics, Virginia Polytechnic Institute and State University, \\ Blacksburg, Virginia 24061 \\ (Received 24 August 1975)

\begin{abstract}
Recent investigations have utilized the measurement of the critical angle for reflection from a liquid-solid interface for determination of the elastic constants of the solid. For anisotropic media, this technique is appropriate only for certain special cases of the incident plane and reflecting surface. We discuss here the general condition for the critical angle in anisotropic media and show that for some planes in quartz, major errors may arise if one employs the ususl statement of Snell's law for definition of the critical angle.
\end{abstract}

Subject Classification: [43] 20.30; [43] 35.26.

Recent investigators have utilized measurements of the critical angle for reflection from a liquid-solid interface to measure phase velocities along the surface (see, for example, Refs. 1-3) and suggested that these velocities could then be used to determine the elastic constants. In addition, Diachok et al. ${ }^{4}$ have determined the orientation of a quartz single crystal by determining the critical angles. In the experiments reported by these authors, the technique used was quite appropriate and led to results close to those predicted because of fortuitous selection of the reflection surfaces and incident planes. We wish to point out here, however, that this technique can not be used in general for anisotropic solids, and, indeed, may lead to rather large errors if one does try to apply it without prior knowledge of the slowness surface for the solid.

In the isotropic case, Snell's law of refraction states that the phase velocities of the incident and refracted waves are related by the expression $v_{I} / \sin \theta_{I}=v_{R} / \sin \theta_{R}$, where $\theta_{I}$ and $\theta_{R}$ are the angles between the incident and reflected wave vectors and the normal to the reflecting surface, respectively. Then, the critical angle of incidence, $\theta_{c}$, is assumed to occur when $\theta_{R}$ becomes equal to $90^{\circ}$, and hence by this definition, the critical angle is given by

$$
\sin \theta_{c}=v_{I} / v_{R} \text {. }
$$

Since the direction of the refracted wave at the critical angle is assumed to correspond to the direction of a body wave propagating along the boundary, Eq. 1 can be used to calculate $v_{R}$ for this known direction and a sufficient number of independent measurements will yield data from which the elastic constants can be determined.

It has been recently pointed out, ${ }^{5}$ however, that, in general, the critical angle occurs when the energy flux associated with the refracted wave becomes parallel to the interface of the two media and not when $\theta_{R}=90^{\circ}$. For this general definition of the critical angle, the governing equations have too many unknowns to allow for determination of the elastic constants by the critical angle experimental technique unless one has prior knowledge of the slowness surface of the solid. The slowness surface, of course, depends in part upon the elastic constants. One is thus caught in a dichotomy.

Thus, consider the dot product of the group velocity ${ }^{6}$ $\mathrm{g}$ and the vector $\mathbf{b}$ which is the vector component of all slowness vectors satisfying the reflecting-refraction problem on the interface, (see, for example, Fig. 1 in Ref. 5). Since b can be written in terms of the refracted wave as $\mathrm{m}_{R}-z_{R} \nu$, we have

$$
\mathrm{b} \cdot \mathrm{g}=\mathrm{m}_{R} \cdot \mathrm{g}-z_{R} \nu \cdot \mathrm{g}=1-z_{R} \nu \cdot \mathrm{g} \text {. }
$$

The last step follows from the fact that the slowness vector $\mathrm{m}_{R}=\mathrm{n}_{R} / v_{R}$, where $\mathrm{n}_{R}$ is the wave normal of the refracted wave, and from the fact that $g \cdot \mathrm{n}_{R}=v_{R} \cdot{ }^{7}$ Now when the critical angle occurs, according to the general definition given above, $\mathrm{g}$ becomes perpendicular to the interface normal $\nu$; and, therefore, at the critical angle of incidence, we have

$$
\mathrm{b}_{c} \cdot \mathrm{g}_{c}=1 \text {. }
$$

The vector $b$ is determined by the incident wave and, in fact, $\left|\mathrm{b}_{c}\right|=\sin \theta_{c} / v_{I_{c}}$. Thus, Eq. 3 can be rewritten in the form

$$
g_{c} \sin \theta_{c} \cos \beta_{c}=v_{I_{c}},
$$

where $\beta$ is the angle between $b$ and $g$ and the subscript $c$ indicates all values are evaluated at the critical angle. Finally, we can write Eq. (4) in terms of the phase velocity of the refracted wave by again making use of the relationship $\mathrm{g} \cdot \mathrm{m}_{R}=1=g \cos \theta / v_{R}$, where $\theta$ is the angle between the normal to the refracted wave and its associated energy flux. Evaluating these quantities at the critical angle of incidence, we have the final general expression for the critical angle:

$$
\sin \theta_{c}=\frac{v_{I_{c}}}{v_{R_{c}}} \frac{\cos \theta_{c}}{\cos \beta_{c}} .
$$

Again, for emphasis, we point out that in Eq. 5, $v_{R_{c}}$ is not generally the phase velocity of a body wave propagating in a direction parallel to the interface between the two media; but rather, it is the phase velocity of a wave which has its associated energy-flux vector parallel to the interface. This wave normal makes an angle $\phi$ with its energy flux. While the vectors $b, m_{R}$, and $\nu$ must all be coplanar, $E$ generally will not lie in this plane (the incident plane), and hence $\phi$ is not simply the angle between $m_{R}$ and the interface. Thus, in general, measurement of the critical angle $\theta_{c}$ will not be sufficient to determine $v_{R_{c}}$ and hence neither the elastic constants nor the crystal orientation. The angles $\theta_{c}$ and $\phi_{c}$ 


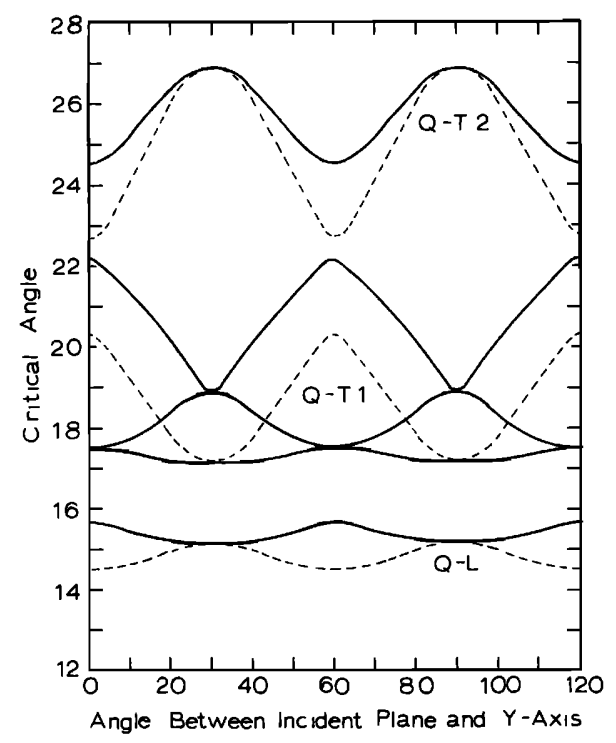

FIG. 1. Critical angle of incident, quasilongitudinal, and quasitransverse modes, versus incident angle for the classical Snell's law (dotted lines) and for the modified law given in the text (solid lines). ${ }^{8}$

must also be known, and these are related in a complex fashion to the elastic constants through the slowness surface of the solid.

Figure 1 presents the results of numerical calculations performed using the techniques discussed in Refs. 5 and 8 for the critical angle at a plane interface between water and quartz. For these calculations, the $x y$ plane of quartz was fixed as the interface and the plane of incidence was varied by rotation about the $z$ axis. The critical angle was then determined for each of the three possible refracted modes in quartz for an incident longitudinal wave in the water. In Fig. 1 the dotted curves give the results based upon the classical expression of Snell's law while the solid curves delineate the results obtained on the basis of the new expression given in Eq. 5. It is obvious from Fig. 1 that large errors may arise in measurement of the critical angle for certain incident planes and subsequent evaluation of the elastic constants. As previously stated, one can, with some effort, obtain the curves of Fig. 1 when the elastic constants are already known, but using the critical angle technique to calculate the elastic constants is impossible if one considers the full statement of Snell's law in Eq. 5.

It is also of interest to note in Fig. 1 that while there are indeed only three permitted body modes in quartz, in general five refracted waves are possible, leading to five distinct critical angles. This fact is due to the reentrant contours of the slowness surface for the quasitransverse mode 1 (Ref. 8). One should use care, however, in applying Fig. 1 too freely to experimental results. No calculations have as yet been made to determine the refraction coefficients. It is possible that some of these modes have zero refraction coefficients for certain incident planes, and hence would never evidence themselves at the calculated critical angles.

This is known to occur for the $y z$ incident plane. However, for other incident planes, preliminary calculations do indicate that all five modes are generally refracted.

*Currently at Bettis Atomic Power Laboratory, West Mifflin, Pennsyl vania.

${ }^{1} \mathrm{H}$. Engan, K. A. Ingerbrigtsen, and A. Tonning, Appl. Phys. Lett. 10, 311-313 (1967).

${ }^{2}$ L. S. Fountain, J. Acoustic Soc. Am. 42, 242-247 (1967).

${ }^{3}$ F. R. Rollins, Jr., T. C. Lim, and G. W. Farnell, Appl. Phys. Lett. 12, 236-238 (1970).

${ }^{4}$ O. I. Diachok, R. J. Hallermier, and W. G. Mayer, Appl. Phys. Lett. 17, 288-289 (1970).

${ }^{5}$ E. G. Henneke II, J. Acoust. Soc. Am. 51, 210-217 (1972). ${ }^{6} \mathrm{The}$ group velocity is related to the energy flux by a scalar factor. Thus $\mathrm{g}=1 / \epsilon \mathrm{E}$, where $\epsilon$ is the total energy density.

${ }^{7}$ F. I. Fedorov, Theory of Elastic Waves in Crystals (Plenum, New York, 1958).

${ }^{8}$ G. L. Jones and E. G. Henneke II, IEE E Trans. Sonics Ultrașon. SU-20, 267-274 (1973). 